\title{
ARTÍCULO ORIGINAL \\ Metodologías y materiales para el \\ entrenamiento en técnicas quirúrgicas: revisión sistemática
}

\section{Methodologies and materials for training in surgical techniques: systematic review / Metodologias e materiais para treinamento em técnicas cirúrgicas: uma revisão sistemática}

Sebastián Calle-Díaz ${ }^{1}$, Sofía García-Osorio ${ }^{2}$, José F. Flórez-Arango ${ }^{1,3}$, Ever A. Torres-Silva ${ }^{1}$, Iván F. Luna-Gómez ${ }^{1}$, Sandra M. Fernández-Ramírez ${ }^{1}$

\section{RESUMEN}

Objetivo: Revisar las metodologías y los materiales utilizados en la enseñanza de técnicas quirúrgicas para personal de la salud, publicados durante los últimos siete años. Metodología: Se realizó una revisión bibliográfica en la base de datos PubMed sobre materiales y metodologías utilizados en educación quirúrgica determinando criterios de inclusión y exclusión para filtrar los diferentes artículos.

Resultados: Se encontraron 1190 artículos, de los cuales se descartaron 826 por no cumplir con el rango de la revisión sistemática.

Se utilizaron 59 artículos que cumplieron los criterios de inclusión y de exclusión para la revisión.

Conclusiones: El entrenamiento para obtener habilidades en técnicas quirúrgicas a través de la simulación es una oportunidad para estudiantes y maestros de avanzar en el saber y el hacer; se deben acoplar los materiales con el espacio para obtener resultados esperados. La investigación continúa con el fin de encontrar mejores opciones para la adquisición de las destrezas y habilidades quirúrgicas.

Palabras clave: simulación; entrenamiento simulado; cirugía general; técnicas de diagnóstico quirúrgico

\section{ABSTRACT}

Objective: To review the methodologies and materials used in the teaching of surgical techniques for health personnel, which have been published over the last seven years. Methodology: A literature review was carried out in the PubMed database on materials and methodologies used in surgical education, based on inclusion and exclusion criteria to filter the different articles.

Results: A total of 1190 articles were found, of which 826 were discarded for not meeting the range of the systematic review. 59 articles that did meet the inclusion and exclusion criteria were used for the review.

Conclusions: Training to obtain skills in surgical techniques through simulation is an opportunity for students and teachers to advance in knowing and doing; materials must be matched with the circumstances to obtain expected results. New research must be carried out in order to find better options for the acquisition of surgical skills and abilities. Keywords: simulation; simulation training; general surgery; diagnostic techniques surgical

\section{RESUMO}

Objetivo: Revisar as metodologias e materiais utilizados no ensino de técnicas cirúrgicas para profissionais de saúde, publicados nos últimos sete anos.
Fecha de recibido:

7 de mayo de 2019.

Fecha de aprobación: 7 de julio de 2020.

Forma de citar este artículo: Calle-Díaz S, García-Osorio S, Flórez-Arango JF, TorresSilva EA, Luna-Gómez IF, Fernández-Ramírez SM. Metodologías y materiales para el entrenamiento en técnicas quirúrgicas: revisión sistemática. Med UPB. 2021;40(1):35-45. DOI:10.18566/medupb.v40n1.a06

1 Universidad Pontificia Bolivariana. Medellín, Colombia.

2 Corporación Universitaria Remington. Medellín, Colombia.

3 Universidad de Texas A \& M. Texas, EE. UU.

\section{Dirección de}

correspondencia: Iván Felipe Luna Gómez. Correo electrónico: ivan.luna@upb. edu.co 
Metodologia: Foi realizada uma revisão bibliográfica na base de dados PubMed sobre materiais e metodologias utilizadas na educação cirúrgica, determinando critérios de inclusão e exclusão para filtrar os diferentes artigos.

Resultados: foram encontrados 1190 artigos, dos quais 826 foram descartados por não atenderem à abrangência da revisão sistemática. 59 artigos que atenderam aos critérios de inclusão e exclusão foram utilizados para a revisão.

Conclusões: $O$ treinamento para obtenção de habilidades em técnicas cirúrgicas por meio de simulação é uma oportunidade para alunos e professores avançarem no saber e no fazer; os materiais devem ser combinados com a lacuna para obter os resultados esperados. A pesquisa continua a fim de encontrar melhores opções para a aquisição de habilidades e habilidades cirúrgicas.

Palavras-chave: simulação; treinamento simulado; cirurgia geral; técnicas de diagnóstico cirúrgico

\section{INTRODUCCIÓN}

La palabra cirugía proviene del griego cheirourgía. Cheiro significa mano, urgia o ergon trabajo; cirugía es trabajo manual. Implica la manipulación mecánica de las estructuras anatómicas de un ser humano con fines médicos ${ }^{1}$.

Las maniobras quirúrgicas básicas que un médico aprende durante la carrera implican la adquisición de destrezas manuales de tipo cruentas, es decir, que causan derramamiento de sangre. Estos procedimientos, por su carácter de invasivos, implican una dificultad en el proceso de enseñanza tradicional realizada al lado y sobre el paciente ${ }^{2}$; como consecuencia de ello nace una iniciativa mundial que busca mejorar las condiciones de la educación en técnicas quirúrgicas por medio de la simulación. La simulación se define como una técnica para reemplazar o amplificar experiencias reales con experiencias guiadas, a menudo inmersivas en la naturaleza, que replican aspectos sustanciales del mundo real de una manera totalmente interactiva ${ }^{3}$. En el área de la salud, consiste en situar a un estudiante en un contexto que imite algún aspecto de la realidad clínica y en medicina ha sido utilizada para reproducir experiencias reales de pacientes a través de escenarios adecuadamente guiados y controlados.

La simulación es un método de entrenamiento emergente que incorpora los tres pilares de la educación y se ha demostrado que mejoran el rendimiento del cirujano. Muchos estudios han evidenciado que la simulación y la práctica fuera de la sala de operaciones tiene un efecto positivo en adquisición de habilidades ${ }^{4}$.

La simulación se usó por primera vez cuando se utilizaron modelos de hojas y arcilla para simular la primera operación registrada, una reconstrucción nasal frontal en la India antigua en 600 a.C C $^{3}$. Desde el cambio del siglo XX al siglo XXI, la educación basada en la simulación y la formación se ha convertido en un tema importante de investigación. Desde 2000, las 10 revistas de mayor impacto quirúrgico han publicado más de 350 artículos en los que la simulación es una metodología importante ${ }^{5,6}$.

El entrenamiento quirúrgico tradicional expone a los pacientes a riesgos, particularmente cuando se aplican nuevas tecnologías a operaciones complejas. La disminución de las tasas de natalidad, las restricciones de horas de trabajo y la creciente necesidad de habilidades quirúrgicas especializadas contribuyen a reducir las oportunidades educativas para los aprendices de cirugía ${ }^{6-8}$.

La reducción de tiempo en las prácticas clínicas reales y los límites clínicos establecidos por ética han llevado a que se realicen experimentos con el fin de encontrar materiales y metodologías aplicables en el entrenamiento quirúrgico. Se ha demostrado que la capacitación en simulación mejora el estado de los pacientes y es un valioso complemento al método tradicional de capacitación de cirujanos ${ }^{6,9-11}$. La simulación permite la práctica repetida en un entorno controlado por mentores; estos espacios son pensados para los aprendices 
y adaptados a sus necesidades. El estudiante tiene la posibilidad de estar en escenarios comunes, comunicar de manera clara y apropiada la información, estar en situaciones estresantes en las que el cirujano debe manejar sus propias emociones y contar con la confianza requerida para entregar al paciente un apropiado cuidado ${ }^{8,12}$. Los estudiantes de cirugía valoran la importancia de las prácticas en simulación y relatan la necesidad de talleres de procedimientos basados en planes de estudios de habilidades quirúrgicas mediados por simulación ${ }^{13,14}$.

Por consiguiente, la simulación consiste en recrear un entorno clínico haciendo uso de materiales, insumos, equipos y simuladores, por ello es importante distinguir entre simulador y simulación. Por simulador se entiende un maniquí, programa o equipo utilizado en la enseñanza, sin embargo, la simulación va más allá del equipo y tiene que ver con la recreación completa del entorno. Un adecuado engranaje entre lo simulado y el entorno permitirá que el aprendiz consiga una experiencia más real. Los avances tecnológicos a lo largo del tiempo han llevado al desarrollo de diversos simuladores considerados un método eficaz para adquirir conocimiento en técnicas quirúrgicas, los cuales se han ido introduciendo en el mercado y entorno educativo. La complejidad, la fidelidad y las funciones de los simuladores disponibles varían y, según los recursos, se debe seleccionar el simulador apropiado para la habilidad que se requiere enseñar ${ }^{15-20}$.

Existen diferentes tipos de simulación, estas son algunas definiciones ${ }^{15-21}$ :

- Simulación de cuidado de la salud con tecnología mejorada

Grupo de materiales y dispositivos creados o adaptados para capacitar a profesionales de la salud en un entorno simulado. Incluyen simuladores de realidad virtual basados en computadora, maniquíes estáticos de alta fidelidad, modelos de plástico, animales vivos, productos de animales inertes y cadáveres humanos.

- Simulación basada en computadores

Esta actividad de aprendizaje está diseñada para proporcionar una experiencia con entradas y salidas exclusivamente confinadas a computadores.

- Juego de roles

Se asumen actitudes, acciones y discurso de otro en un esfuerzo por comprender un punto de vista diferente. Por ejemplo: a los estudiantes de Medicina se les da la oportunidad de interpretar a un paciente o un cirujano.

- Realidad virtual

Simulaciones que utilizan una variedad de características 3D inmersivas y altamente visuales para replicar situaciones de la vida real o procedimientos de atención médica; la simulación de realidad virtual se distingue de la simulación basada en computadores porque generalmente incorpora interfaces físicas o dispositivos hápticos.
La educación ha visto una gran mejora con la tecnología tridimensional (3D) de modelos virtuales en ciencias anatómicas. Se han utilizado varios datos para crear estos modelos que han ido mejorando constantemente ${ }^{21}$. Los estudios han demostrado que los modelos con sistemas de proyección virtual 3D pueden mejorar el aprendizaje de las estructuras anatómicas en los estudiantes y que ellos prefieren este método de enseñanza. Sin embargo, el sistema de entrenamiento quirúrgico basado en realidad virtual no se ha estudiado completamente para lograr tiempo real y realismo; todavía hay problemas que abordar en este campo ${ }^{16-20}$. Este estudio plantea como objetivo realizar una revisión sistemática de las metodologías y los materiales utilizados en la enseñanza de técnicas quirúrgicas para personal de la salud, publicados durante los últimos siete años.

\section{METODOLOGÍA}

Con el propósito de obtener resultados concretos a través de los criterios de inclusión y exclusión, filtrarlos y escribir un artículo de revisión pertinente, se eligió la base de datos para realizar la búsqueda y se crearon las ecuaciones de búsqueda con vocabulario $\mathrm{MeSH}$ y Emtree.

La ecuación de búsqueda se diseñó utilizando operadores booleanos (AND/OR), así: ("Motor skills"[Mesh] OR "Teaching Materials"[Mesh] OR "Simulation Training”[Mesh]) AND “General Surgery"[Mesh] para PubMed; además se aplicó un filtro de año de publicación a partir de 2013 hasta 2020, es decir, artículos que no tuvieran una fecha de publicación superior a los siete años. La búsqueda se limitó a estudios de metodologías y materiales empleados en el entrenamiento para técnicas quirúrgicas, haciendo énfasis en cirugía general.

Se obtuvieron 1190 resultados, de los que se eligieron 364 que aplicaban en el rango de tiempo para ser evaluados; el primer filtro fue realizado por uno de los autores mediante a) revisión de los títulos de los artículos, b) descarte de referencias duplicadas, c) eliminación de artículos sin resumen, d) exclusión de estudios en idiomas diferentes a inglés y español y e) descarte de artículos que no están relacionados con entrenamiento en técnicas quirúrgicas basadas en simulación. Una vez obtenido el resultado del primer filtro, se realizó la revisión del resumen por tres de los autores con los siguientes criterios de inclusión: a) estudios que contengan metodologías para la educación en técnicas quirúrgicas y b) estudios que incluyan dentro de su desarrollo materiales para ser empleados en el aprendizaje de técnicas quirúrgicas.

Los criterios de inclusión y exclusión se resolvieron por consenso entre los autores. Se obtuvieron los resultados consignados en la Tabla 1 y se inició con la escritura del artículo de revisión. Una limitante que se evidenció al 
Tabla 1. Resultados de las ecuaciones de búsqueda.

\begin{tabular}{ll}
\hline Base de datos & Artículos encontrados \\
\hline PubMed & Se encontraron 1190 artículos, de los que se descartaron 826 por estar fuera del rango \\
de los últimos siete años. Quedaron 364, de los que, de acuerdo con los criterios de \\
inclusión y exclusión, se utilizaron 59.
\end{tabular}

Figura 1. Flujograma de selección de artículos.

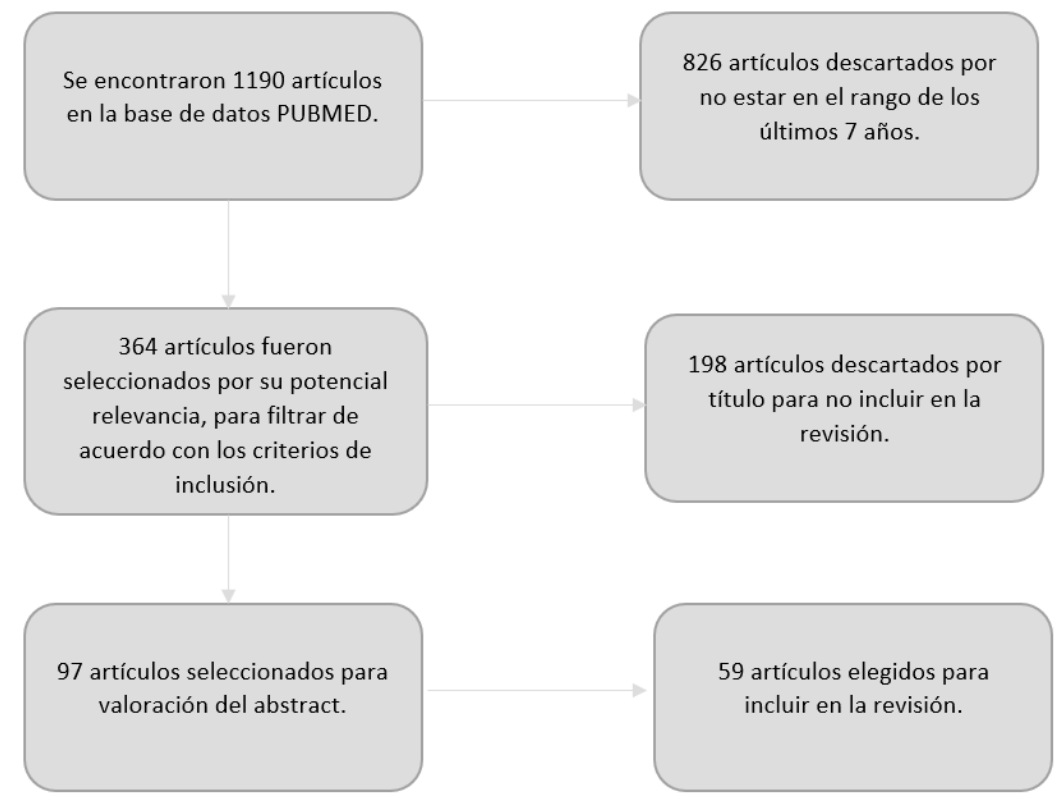

seleccionar los artículos fue la imposibilidad de contar con búsqueda en la base de datos Embase, debido a que los autores no tienen acceso autorizado.

\section{RESULTADOS}

Para la educación en técnicas quirúrgicas, se utilizan herramientas de apoyo, como organismos vivos, animales, pacientes reales, incluyendo sus partes y material sintético; además, se cuenta con simuladores de baja hasta alta fidelidad. Asimismo, la simulación incluye el juego de roles entre estudiantes y maestros, de esta manera el aprendiz en determinado momento podrá ser paciente o cirujano; sin embargo, a pesar de las restricciones surgidas con el tiempo, aún se realizan prácticas quirúrgicas en cadáveres ${ }^{9}$.

La simulación es ampliamente aceptada e integrada en la capacitación médica, desde el pregrado hasta la educación médica continua. Además, existe una perspectiva más amplia de las aplicaciones de la simulación en todo el cuidado de la salud. La capacitación basada en simulación ha ayudado a descubrir la importancia de la ingeniería y de los factores humanos en el diseño de sistemas que facilitan los comportamientos o resultados deseados y que desalientan activamente las acciones inseguras o inapropiadas ${ }^{10}$.

\section{Simulación de cuidado de la salud con tecnología mejorada}

La práctica médica ha ido evolucionando, y a los conocimientos adquiridos en animales vivos, productos de animales inertes y cadáveres humanos, ahora se suman simuladores estáticos de alta fidelidad y modelos de plástico, que permiten una interacción real del estudiante con un episodio clínico específico. Estos se han convertido en una necesidad, ya que algunos estudios han demostrado que solo el entrenamiento motor adicional o de conciencia no es suficiente para potenciar el entrenamiento usual ${ }^{11}$.

En esta era tecnológica, todos los sectores han decidido avanzar a la par de la evolución, un ejemplo es el Departamento de Cirugía de la Universidad de Wisconsin, donde desarrollaron un modelo de tumor pélvico que 
simula sangrado inesperado, con el fin de evaluar el desempeño del robot llamado Da Vinci, desarrollado para el entrenamiento quirúrgico. Además, se evalúa la habilidad de los estudiantes al usarlo. En un estudio los alumnos enfrentaron un sangrado inesperado, a quienes se les evaluó la reacción frente a este ${ }^{12}$.

Ahora bien, la simulación puede ser variada y dar los mismos resultados, esto se evidencia en un estudio en la Universidad de Pensilvania, donde compararon los resultados de entrenamiento de residentes en la aplicación de un catéter venoso central en la yugular interna, mediante simulador de alta fidelidad o en el entrenador robótico háptico dinámico (DHRT, por sus siglas en inglés), los resultados no fueron diferentes, por lo que ambos métodos son válidos. Otro ejemplo del avance tecnológico son los simuladores de cirugía laparoscópica con retroalimentación sensorial "háptica", entre los que se identifica un novedoso simulador de funduplicatura laparoscópica con un sistema de validación de habilidades quirúrgicas, que desarrollaron a partir de un modelo de cuerpo infantil de un año basado en datos tomográficos y un modelo de neumoperitoneo reproducido en función de la situación clínica ${ }^{13}$.

El ultrasonido es otro campo donde ha intervenido la simulación, por ejemplo, se prueban los efectos del entrenamiento previo sobre el rendimiento de los participantes. En este estudio se construyeron tres modelos de objetivos ecogénicos con clavijas y bolas de madera. Estos modelos se pusieron en un cubo de agua cubierto con una hoja de gelatina balística que representa la piel; el modelo demostró su validez ${ }^{14}$.

La colonoscopia también se ha beneficiado de este método; a los residentes de AimSurgery se les evaluó la capacidad de respuesta ante el entrenamiento de colonoscopia simulada. Fueron sometidos a capacitación en un modelo físico que incluye colonoscopia, bandejas sintéticas y un simulador híbrido. El entrenamiento con colonoscopia simulada en un modelo físico de bajo costo mejoró el desempeño de los residentes de cirugía con menor tiempo de procedimiento, mayores tasas de colonoscopia completa y una vista apropiada ${ }^{15}$.

Además, no solo existe un interés particular en diseñar simuladores basados en robots, también se pretende desarrollar prototipos de modelos humanos más fieles a la realidad, por ejemplo, el centro de simulación de la Universidad Northwestern busca crear y validar una simulación de alta fidelidad mediante el uso de tejido anatómico bovino, para la reparación toracoscópica de atresia esofágica y fístula traqueoesofágica a partir de una reproducción a escala de una caja torácica neonatal ${ }^{16}$.

Por otro lado, en Austria y Alemania han realizado prototipos de hueso para practicar inserción ósea. Los levantamientos de injertos parietales se entrenan habitualmente en muestras humanas o animales o se realizan directamente en pacientes sin entrenamiento extensivo, y el cirujano necesita aplicar las fuerzas apropiadas a las herramientas de rotación rápida. Esto requiere experiencia y entrenamiento, por tanto, en este estudio se evaluaron materiales sustitutos óseos con el objetivo de proporcionar un cráneo artificial anatómicamente correcto $^{17}$. Además, existe el desarrollo de hueso temporal sintético que se imprimió en materiales compuestos con tecnología de impresión tridimensional, con el que se evaluaron las experiencias de los aprendices analizando cuatro dominios: realismo anatómico, utilidad como herramienta de entrenamiento, utilidad basada en tareas y reacciones generales. Las respuestas en todos los dominios indicaron un alto grado de aceptación, lo que sugiere que el hueso temporal impreso tridimensional es una herramienta útil en el desarrollo de habilidades ${ }^{18}$.

La simulación es apropiada para practicar cirugías de cavidades pequeñas, por ejemplo, la cirugía de paladar hendido, difícil para los cirujanos debido a que deben trabajar en una cavidad pequeña, de poca profundidad, acceso limitado, mala visualización, tejidos delicados, disección muscular y sutura en profundidad. Por ende, se creó el simulador de paladar hendido más simple para facilitar la formación de cirujanos. Este modelo fue hecho con componentes fácilmente disponibles, como esponja almohadilla, yeso adhesivo, botella plástica y grapadora ${ }^{19}$.

Para el caso de la cirugía vascular, desarrollaron un modelo porcino para practicar anastomosis aórtica. E1 informe describe un nuevo simulador que utiliza cerdos con bypass cardiopulmonar y validación mediante un ensayo controlado con residentes de cirugía general que aprenden anastomosis aórtica. Se evaluó este simulador con la siguiente hipótesis: el simulador perfundido porcino es tan bueno o mejor que modelos de baja fidelidad utilizados para el entrenamiento de la anastomosis vascular ${ }^{20}$.

Los modelos porcinos han sido usados de otros modos. Ha sido empleado material específico para prácticas de trasplante, lo que solventa el problema de la escasez de órganos. En Kokugakuin University Tochigi Junior College (Japón), utilizaron modelos porcinos para este tipo de procedimientos, que, además de comprensión de la anatomía compleja del órgano, requiere alto nivel de habilidad quirúrgica. Por tanto, los cirujanos deben dominar la técnica quirúrgica. Tanto el entrenamiento como la simulación quirúrgica que utiliza un modelo animal son útiles desde este punto de vista educativo, en comparación con el entrenamiento en el trabajo. En ese mismo sentido, un programa con tejidos no vivos sirve para el aprendizaje y mejoramiento de las capacidades en microcirugía en un modelo ex vivo de pollos ${ }^{21}$.

Se requiere la motivación para que los estudiantes utilicen simuladores. Una de las estrategias es establecer competiciones entre ellos ya que algunos simuladores 
permiten cuantificar los movimientos y actividades. Este proceso de simulación médica reafirma la confianza del cirujano. El departamento de cirugía de la Universidad de Carolina del Norte aprovechó el entrenamiento con robot para incrementar la adquisición de habilidades de los residentes quirúrgicos que tienen presión del tiempo e imperativos financieros para adquirirlas de otro modo. En este sentido, la cirugía robótica ofrece ventajas, como la imagen tridimensional con visibilidad mejorada, amplitud de movimientos quirúrgicos a través de los brazos robóticos y ergonomía mejorada. Estas características han alentado la aceptación de la plataforma robótica en Estados Unidos. Sin embargo, no solo se necesitan grandes robots, sino que también se requieren modelos anatómicos para realizar procedimientos, por ejemplo, existe un modelo para disección rectal con el robot $\mathrm{Da}$ Vinci, con excelentes resultados en tiempo quirúrgico y habilidad ${ }^{22,23}$.

\section{Simulación basada en computadores}

La educación tradicional consiste en conferencias didácticas por un docente y prácticas clínicas, que se complementa con el aprendizaje de libro y documentos. La sociedad ha ido evolucionando, hasta tal punto que las plataformas de medios electrónicos están reemplazando los medios impresos y la educación médica se ha adaptado a este cambio. Según la Escuela de Medicina de la Universidad Stanford, el aprendizaje móvil es eficiente e innovador, y tiene la capacidad de acceder a la información de alta calidad en cualquier lugar y en cualquier momento. En respuesta, muchas escuelas de medicina han cambiado el uso de métodos pedagógicos hacia la incorporación de métodos más andrográficos. En un estudio se demostró que el $88.4 \%$ de los estudiantes utilizó un teléfono inteligente con fines académicos, incluido el 98.1\% de los encuestados de áreas quirúrgicas ${ }^{24}$.

Los principios del aprendizaje en adultos son aprendizaje centrado en un problema, aprendizaje en un entorno de apoyo, aprendizaje orientado a la experiencia, retroalimentación adquirida y aprendizaje activo ${ }^{25}$. En la simulación basada en computadores el aprendizaje está diseñado para proporcionar una experiencia con entradas y salidas confinadas a computadores u otro dispositivo de asistencia que cumplen los cinco principios mencionados.

En esta revisión se encuentra un método simple en el cual se evidencia este tipo de simulación. Consta de un aplicativo móvil desarrollado para residentes de cirugía, esta aplicación envía aleatoriamente preguntas diarias de un banco, una alarma de notificación y una retroalimentación inmediata con información importante sobre el tema tratado. El uso de esta novedosa aplicación demostró la mejor la participación en comparación con formato de correo electrónico ${ }^{26}$.

\section{Realidad virtual}

La ingeniería biomédica ha desarrollado simuladores de cirugía laparoscópica con elementos comerciales como el Phantom Omni validados por personas con diferentes niveles de experticia para lograr un producto cercano al mercado (eLap4D) ${ }^{6}$. Otros dispositivos comerciales como el Leap Motion permiten la simulación de cirugías mediante gestos en las manos, aunque con limitantes en cuanto a la complejidad de los diferentes gestos de la mano y variables como la fuerza y tiempos de reacción ${ }^{27}$. Este tipo de desarrollos requieren validación en cuanto la correlación entre la sensación háptica y las habilidades motoras $^{28}$, ya que se han demostrado diferencias significativas en los parámetros del simulador según lo use un novato o un experto ${ }^{29}$. Todo esto es importante ya que la cirugía asistida por robot ha surgido como una alternativa mínimamente invasiva para los procedimientos ginecológicos como histerectomía, miomectomía, estadificación del cáncer y reparación de prolapso. El enfoque robótico ofrece mayor destreza con los instrumentos laparoscópicos de muñeca, pero también requiere entrenamiento técnico exclusivo de la cirugía robótica ${ }^{30}$.

Otro aspecto importante para la realidad virtual es la posibilidad de crear entornos para una adaptación mejor en el escenario real. Un caso es el desarrollo de un entorno para facilitar a estudiantes de enfermería y medicina la primera visita al quirófano, que reduce el miedo a cometer errores y la propensión a cometerlos, y mejora el comportamiento y la actitud en el espacio con los colaboradores y pacientes ${ }^{31}$.

Por último, de acuerdo con los resultados de búsqueda solo hay un artículo que hace referencia a la realidad aumentada combinada con sensórica y materiales con memoria de forma, como el nitinol, como prueba de concepto para el desarrollo de un simulador de colecistectomía completo ${ }^{32}$.

A continuación, se hace una relación con las correspondientes referencias de los artículos que nutren esta revisión sistemática. Se incluye el tipo de simulación y destrezas que se fortalecen (Tabla 2).

\section{DISCUSIÓN}

El entrenamiento basado en la simulación ha sido empleado por los cirujanos durante mucho tiempo para enseñar sus habilidades a los residentes y estudiantes. Esto permite evaluar el desempeño fuera de la sala de operaciones en un entorno seguro y de bajo riesgo, con la oportunidad de realizar comentarios constructivos y prácticas deliberadas ${ }^{33}$. Los educadores quirúrgicos han incorporado simuladores de técnicas básicas y promueven los laboratorios en el currículo educativo de estudiantes de Medicina para enseñar habilidades y procedimientos 
Tabla 2. Tipo de simulación y destrezas que se fortalecen.

\begin{tabular}{|c|c|c|c|c|c|c|c|}
\hline Artículo & Animal & Sintética & Computacional & $\begin{array}{c}\text { Realidad } \\
\text { virtual aumentada }\end{array}$ & $\begin{array}{c}\text { Destrezas } \\
\text { físicas }\end{array}$ & $\begin{array}{l}\text { Destrezas } \\
\text { cognitivas }\end{array}$ & $\begin{array}{c}\text { Destrezas } \\
\text { comunicacionales }\end{array}$ \\
\hline Porras et al. ${ }^{1}$ & & & & & $x$ & $\mathrm{x}$ & $x$ \\
\hline Torres et al. ${ }^{2}$ & $x$ & & & & $x$ & & \\
\hline Agha et al. ${ }^{3}$ & & $x$ & & & & $x$ & \\
\hline Alicia et al. ${ }^{4}$ & & & & & $x$ & $x$ & \\
\hline Johnston et al. ${ }^{5}$ & $x$ & $x$ & $x$ & $x$ & $x$ & $x$ & $x$ \\
\hline Ebbe Thinggaard et al. ${ }^{6}$ & & $x$ & & & $x$ & & \\
\hline Barsness et al. ${ }^{7}$ & & & & & & $x$ & $x$ \\
\hline Breaud et al. ${ }^{8}$ & & $x$ & & & & $x$ & $x$ \\
\hline Hassan et al. ${ }^{9}$ & $x$ & & & & $x$ & & \\
\hline Brown et al. ${ }^{10}$ & & & & & & $x$ & \\
\hline Causby et al. ${ }^{11}$ & & & $x$ & & $x$ & & \\
\hline Walker et al. ${ }^{12}$ & & $x$ & & & $x$ & & \\
\hline Jimbo et al. ${ }^{13}$ & & $x$ & $x$ & & & & \\
\hline Shariff et al. ${ }^{14}$ & & $x$ & & & $x$ & $x$ & \\
\hline Buscaglia al. ${ }^{15}$ & & $x$ & & & $x$ & & \\
\hline Barsness et al. ${ }^{16}$ & & $x$ & & & $x$ & & \\
\hline Hollensteiner et al. ${ }^{17}$ & & $x$ & & & $x$ & $x$ & \\
\hline Da Cruz et al. ${ }^{18}$ & & $x$ & & & $x$ & & \\
\hline Şentürk et al. ${ }^{19}$ & & $x$ & & & $x$ & & \\
\hline Mitterer et al. ${ }^{20}$ & & & $x$ & & & $x$ & \\
\hline Dongmei et al. ${ }^{21}$ & & & $x$ & & & $x$ & $x$ \\
\hline Greig et al. ${ }^{22}$ & & & & $x$ & $x$ & & \\
\hline Shipper et al. ${ }^{23}$ & $x$ & & & & & $x$ & \\
\hline Franko et al. ${ }^{24}$ & & & $x$ & & & & \\
\hline Goodman et al. ${ }^{25}$ & & & & & & $x$ & $x$ \\
\hline Shaw et al. ${ }^{26}$ & & & & & & $x$ & \\
\hline Strzelczyk et al. ${ }^{27}$ & & & & & & $x$ & \\
\hline Mohamadipanah et al. ${ }^{28}$ & & & $x$ & & $x$ & $x$ & \\
\hline Bjer Bjerrum et al. ${ }^{29}$ & & & & $x$ & $x$ & $x$ & \\
\hline Nazema y Siddiqui ${ }^{30}$ & & & $x$ & & $x$ & $x$ & \\
\hline Blanco et al. ${ }^{31}$ & & & $x$ & & & $x$ & \\
\hline Reisinger et al. ${ }^{32}$ & & & $x$ & & & $x$ & \\
\hline Byrne et al. ${ }^{33}$ & & & & $x$ & $x$ & $x$ & \\
\hline Acton et al. ${ }^{34}$ & & & & & $x$ & $x$ & $x$ \\
\hline Tillou et al. ${ }^{35}$ & & & $x$ & & $x$ & $x$ & \\
\hline Kearney et al. ${ }^{36}$ & & & & & $x$ & $x$ & $x$ \\
\hline Paige et al. ${ }^{37}$ & & & & & & $x$ & $x$ \\
\hline Fried et al. ${ }^{38}$ & & & $x$ & & $x$ & $x$ & \\
\hline Gardner et al. ${ }^{39}$ & & & & & & $x$ & $x$ \\
\hline McKinley et al. ${ }^{40}$ & & & & & $x$ & $x$ & \\
\hline Chauvin 41 & & & & & $x$ & $x$ & $x$ \\
\hline Evans et al. ${ }^{42}$ & & & & & $x$ & $x$ & $x$ \\
\hline Cumin et al. ${ }^{43}$ & $x$ & $x$ & $x$ & $x$ & $x$ & $x$ & $x$ \\
\hline Miyasaka et al. ${ }^{44}$ & & & & & & $x$ & $x$ \\
\hline Sullivan et al $\left.\right|^{4.5}$ & & & $x$ & & & $x$ & \\
\hline Stefanidis et al. ${ }^{46}$ & & & & & $x$ & $x$ & $x$ \\
\hline Pucher et al. ${ }^{47}$ & & & & & $x$ & $x$ & $x$ \\
\hline Pucher et al. ${ }^{48}$ & & $x$ & & & $x$ & $x$ & \\
\hline Evgeniou et al. ${ }^{49}$ & & $x$ & & & $x$ & $x$ & $x$ \\
\hline Davies et al. ${ }^{50}$ & $x$ & $x$ & $x$ & $x$ & $x$ & $x$ & $x$ \\
\hline Raphael et al. ${ }^{51}$ & & & & $x$ & $x$ & & \\
\hline Shuxiang et al. ${ }^{52}$ & & & & $x$ & $x$ & & \\
\hline Chen et al. ${ }^{53}$ & & & $x$ & & $x$ & & \\
\hline Sanada et al. ${ }^{54}$ & $x$ & & & & $x$ & $x$ & \\
\hline Schlottmann et al. ${ }^{55}$ & $x$ & & & & $x$ & & \\
\hline Melich et al..$^{56}$ & $x$ & & & & $x$ & $x$ & \\
\hline Annu $\operatorname{Rev}^{57}$ & & & & $x$ & $x$ & $x$ & \\
\hline Groom et al. ${ }^{58}$ & & $x$ & & & $x$ & & \\
\hline Harley et al. ${ }^{59}$ & & & & & $x$ & & \\
\hline
\end{tabular}


operativos básicos ${ }^{34}$. Este modo de aprendizaje permite la elección de ejercicios para manipular elementos de cirugía robótica en un orden específico y progresivo ${ }^{35}$. Además, los residentes pueden desarrollar un conjunto de ejercicios de entrenamiento simulado en cirugía, incluso sin la disponibilidad de materiales sofisticados y costosos. Sin embargo, la presencia de un tutor y las capacidades específicas de programación parecen necesarias para lograr resultados más satisfactorios.

Estas formas de enseñanza fueron iniciadas por la industria aérea en reacción a los estudios en la década de 1970, que muestran que más del $70 \%$ de los choques involucraron el error humano. En esa área se desarrollaron programas estandarizados, basados en simulación para mejorar el trabajo en equipo entre las aeronaves ${ }^{36}$. En la Universidad de Texas, como en muchas instituciones, han dado un impulso para acelerar los esfuerzos en curso para integrar la simulación en los planes de estudio del personal de la salud ${ }^{37}$.

Los errores médicos son una preocupación en la práctica clínica, lo que sugiere la necesidad de ayudas quirúrgicas para la planificación preoperatoria. No solo son los errores en la práctica, también se evidencia la desventaja que tienen algunos profesionales especializados en diferentes áreas para realizar procedimientos de emergencia, por ejemplo, habilitar la vía área del cuello. Por ello, en Aintree University Hospital (Liverpool), simularon con diferentes especialistas (anestesiólogos, cirujanos de cabeza y cuello, cirujanos generales) este procedimiento, cuyo resultado fue que los anestesiólogos lo realizan más rápido, por lo que las diferentes especialidades deberían practicarlo más. Por esto son necesarios cambios en la educación médica que incluyan simulación con evaluaciones para enseñar habilidades cognitivas, técnicas y de toma de decisiones ${ }^{34,38}$.

Además las prácticas permiten a los estudiantes fortalecer el entrenamiento en interacciones sociales que ayudan a liderar y coordinar acciones para mejorar la efectividad de los equipos de cirugía ${ }^{39}$, amén de trabajar en el desarrollo de la inteligencia emocional e identificación de comportamientos y actitudes en escenarios controlados ${ }^{40}$.

La optimización de recursos tecnológicos, de infraestructura y de personal en la simulación son fundamentales. Por eso es importante una buena alineación entre los objetivos de aprendizaje, las características del docente y del alumno, los simuladores y el objetivo de la actividad o proceso. También es importante identificar el grado de fidelidad requerido al simulación, ya que los estudiantes pueden verse sobrecargados por estímulos innecesarios y tener una capacidad de procesamiento cognitivo insuficiente para utilizar el conocimiento y las experiencias en la adquisición de nuevos aprendizajes ${ }^{41}$.

Las restricciones de tiempo subrayan la necesidad de maximizar las horas de trabajo de los residentes y propor- cionar un entorno de aprendizaje de calidad que cultive y optimice sus habilidades clínicas. Capacitar a cirujanos competentes requiere innovación y modernización de los métodos educativos, con el reconocimiento de que el aula quirúrgica está en transformación constante y la comprensión de los estilos actuales de aprendizaje ${ }^{42}$.

La investigación observacional en el quirófano informa que el trabajo en equipo y la comunicación son necesarios y tiene que ver con la calidad en la atención y el cuidado del paciente. Por esto algunos centros hospitalarios y universidades adoptan la simulación como forma de enseñanza en que plantean escenarios que tienen en cuenta la capacitación a realizar y el medio ambiente ${ }^{43}$. Enseñar en un centro de simulación implica inversión de tiempo por parte de los profesores ${ }^{44}$ que deben retroalimentar sobre el desempeño, para permitirles a los estudiantes reflexionar sobre su toma de decisiones, ajustar sus estrategias o comportamientos y pensar sobre los logros ${ }^{45}$.

La simulación ofrece una oportunidad única para que los estudiantes observen cómo se acercan sus compañeros al manejo del paciente y analicen las formas de abordaje ${ }^{44}$. La simulación también se usa comúnmente para capacitar equipos en el cuidado de la salud ${ }^{43}$. Está probado que la habilidad adquirida en simuladores es transferible a la sala de operaciones y la formación basada en las competencias maximiza este beneficio ${ }^{46}$. Se ha demostrado que se pueden mejorar las habilidades no técnicas, esto a su vez incrementa el rendimiento general, por ejemplo, mediante la delegación adecuada de tareas para reducir las distracciones al médico primario o el efecto que tiene la comunicación de planes de gestión al resto del equipo ${ }^{47}$.

Según David Cumin, el Instituto de Medicina de Estados Unidos (IOM, por su sigla en inglés) pide que los equipos que trabajan juntos se capaciten juntos. Esto sugiere la necesidad de una capacitación combinada de las tres disciplinas principales (los equipos secundarios de enfermería, anestesia y cirugía) que componen un equipo de quirófano. Un estudio sobre entrenamiento de equipos multidisciplinarios identificó dos aspectos de la capacitación que también contribuyeron a su éxito: permitir suficiente tiempo para que los participantes aprendieran y asegurar que el ambiente fuera psicológicamente seguro permitiendo al personal sentir un sentido de igualdad ${ }^{43}$.

Según Cumin, no se está llevando a cabo ninguna investigación que involucre la capacitación de equipos multidisciplinarios basada en la simulación, a pesar de la clara necesidad de tales iniciativas. Las barreras para las iniciativas de estas capacitaciones incluyen el reclutamiento, la fidelidad de los modelos quirúrgicos y el $\operatorname{costo}^{43}$.

La educación basada en simulación aún tiene vacíos en cuanto a la relación médico-paciente. Las investigaciones sugieren que muchos eventos adversos prevenibles pueden atribuirse a errores no técnicos que ocurren dentro 
de un contexto de sala. Las rondas representan el principal punto de interacción entre el paciente y el médico, pero se llevan a cabo sin una capacitación o evaluación formalizada. La sala simulada debe proporcionar un entorno en el que los procesos de atención perioperatoria puedan realizarse de forma segura y realista, permitiendo la evaluación multidisciplinaria y la capacitación de las rondas completas de la sala ${ }^{48}$.

\section{Conclusiones}

La investigación continúa y los avances tecnológicos ofrecen gran cantidad de simuladores y tipos de simulación. Cada tipo de simulación tiene que validarse antes de ser utilizado a fines de entrenamiento. Se debe elegir el simulador apropiado a los objetivos educativos teniendo en cuenta el nivel de conocimiento y competencia de los aprendices.

Para que la capacitación sea efectiva, debe integrarse en un plan de estudios quirúrgico y alinearse con la práctica clínica. El reconocimiento de la simulación como herramienta efectiva para enseñar una variedad de habilidades técnicas y no técnicas a los estudiantes es clave en la transformación del aprendizaje quirúrgico. El entorno clínico es complejo e impredecible, una máquina no puede enseñar a un principiante habilidades de comunicación, profesionalismo y trabajo en equipo, por tanto, la simulación debe reflejar el ambiente clínico y recrear las circunstancias de la práctica real.

Para tener éxito en la educación quirúrgica basada en la simulación, se debe lograr un equilibrio adecuado entre el entorno de simulación y simuladores ${ }^{49}$. Más que una tecnología para el desarrollo de destrezas y habilidades en el ámbito de cirugía es la metodología educativa que se debe implementar para que se cumplan los objetivos tanto técnicos como interprofesionales como la comunicación y el trabajo en equipo.

Cabe destacar que el desarrollo tecnológico en el área de cirugía permite cuantificar los movimientos permitiendo identificar mejoras en el aprendizaje y establecer estrategias de gamificación como competencias. Estas actividades son necesarias para motivar a los estudiantes al uso de la simulación.

Los procedimientos son esenciales para las prácticas de tratamiento quirúrgico; para comprenderlos es mejor intentar realizarlos técnicamente, en el caso de operaciones laparoscópicas ${ }^{50}$.

\section{DECLARACIÓN DE CONFLICTO DE INTERESES}

Los autores declaran no tener ningún conflicto de intereses.

\section{REFERENCIAS}

1. Porras-Hernández JD. Enseñanza y aprendizaje de la cirugía. Investig en Educ Médica 2016;5:261-7.

2. Torres RA, Orban RD, Serra EE, Marecos MC, Vargas L, Deffis LI, et al. Enseñanza de técnicas quirúrgicas básicas en simuladores biológicos: experiencia pedagógica en el pregrado. Educ Médica 2003;6:149-52.

3. Agha RA, Fowler AJ. The role and validity of surgical simulation. Int Surg 2015;100:350-7.

4. Heelan Gladden AA, Conzen KD, Benge MJ, Gralla J, Kennealey PT. A vascular anastomosis simulation can provide a safe and effective environment for resident skills development. J Surg Educ 2018,75:1367-73.

5. Johnston MJ, Paige JT, Aggarwal R, Stefanidis D, Tsuda S, Khajuria A, et al. An overview of research priorities in surgical simulation: what the literature shows has been achieved during the 21st century and what remains. Am J Surg 2016;211:214-25.

6. Thinggaard E, Bjerrum F, Strandbygaard J, Konge L, Gögenur I. A randomised clinical trial of take-home laparoscopic training. Dan Med 2019;66:A5525.

7. Barsness KA. Trends in technical and team simulations: challenging the status quo of surgical training. Semin Pediatr Surg 2015;24(3):130-3.

8. Breaud J, Talon I, Fourcade L, Podevin G, Rod J, Audry G, et al. The National Pediatric Surgery Simulation Program in France: a tool to develop resident training in pediatric surgery. J Pediatr Surg 2019;54:582-586.

9. Hassan S, Eisma R, Malhas A, Soames R, Harry L. Surgical simulation flexor tendon repair using Thiel cadavers: a comparison with formalin embalmed. J Hand Surg Eur Vol 2015;40:246-9.

10. Brown KM, Paige JT. Preface. Surg Clin North Am 2015;95:27-8.

11. Causby RS, Mcdonnell MN, Reed L, Hillier SL. A randomised controlled trial of sensory awareness training and additional motor practice for learning scalpel skills in podiatry students. BMC Med Educ 2016;16:309.

12. Walker JL, Nathwani JN, Mohamadipanah H, Laufer S, Jocewicz FF, Gwillim E, et al. Residents' response to bleeding during a simulated robotic surgery task. J Surg Res 2017;220:385-90.

13. Jimbo T, Leiri S, Obata S, Uemura M, Souzaki R, Matsuoka N, et al. A new innovative laparoscopic fundoplication training simulator with a surgical skill validation system. Surg Endosc Other Interv Tech 2017;31:1688-96.

14. Shariff FU, Deladisma AM, Menard JW, Shewokis PA, Lind DS. Use of a hybrid-abdominal wound simulated patient in the ACS/ASE medical student simulation skills curriculum. Am J Surg 2019;217:250-255.

15. Buscaglia JM, Fakhoury J, Loyal J, Denoya PI, Kazi E, Stein SA, et al. Simulated colonoscopy training using a low-cost physical model improves responsiveness of surgery interns. Color Dis 2015;17:530-5. 
16. Barsness KA, Rooney DM, Davis LM. Collaboration in simulation: the development and initial validation of a novel thoracoscopic neonatal simulator. J Pediatr Surg 2013;48:1232-8.

17. Hollensteiner M, Fürst $D$, Esterer $B$, Augat $P$, Schrödl F, Hunger $S$, et al. Novel bone surrogates for cranial surgery training. J Mech Behav Biomed Mater 2017;72:49-51.

18. Da Cruz MJ, Francis HW. Face and content validation of a novel three-dimensional printed temporal bone for surgical skills development. J Laryngol Otol 2015;129:S23-9.

19. Sentürk S. The simplest cleft palate simulator. J Craniofac Surg 2013;24:1056.

20. Mitterer JO. Ask not what post-secondary education can do for psychology: ask what psychology can do for post-secondary education. Can Psychol 2016;47:57-62.

21. Cui D, Chen J, Meyer E, Yang G. Anatomy visualizations using stereopsis: current methodologies in developing stereoscopic virtual models in anatomical education. Adv Exp Med Biol 2019;1156:49-65.

22. Greig ME, Christopher M. A serious game skills competition increases voluntary usage and proficiency of a virtual reality laparoscopic simulator during first-year surgical residents' simulation curriculum. Surg Endosc 2017;31:1643-50.

23. Shipper ES, Miller SE, Hasty BN, Merrell SB, Lin DT, Lau JN. ScienceDirect Association for Academic Surgery Evaluation of a technical and nontechnical skills curriculum for students entering surgery. J Surg Res 2017;219:92-7.

24. Franko OI, Tirrell TF. Smartphone app use among medical providers in ACGME training programs. J Med Syst 2015;36:3135-9.

25. Goodman LJ, Brueschke EE, Bone RC, Rose WH, Williams EJ, Paul HA. An experiment in medical education: a critical analysis using traditional criteria. JAMA 2014:265:2373-6.

26. Shaw CM, Tan SA. Integration of mobile technology in educational materials improves participation: creation of a novel smartphone application for resident education. J Surg Educ 2015;72:670-3.

27. Strzelczyk J, Bialkowska J, Loba J, Jablkowski M. Rapid growth of liver hemangioma following interferon treatment for hepatitis $C$ in a young woman. Hepatogastroenterology 2016;51:1151-3.

28. Mohamadipanah H, Parthiban C, Nathwani J, Rutherford D, DiMarco S, Pugh C. Can a virtual reality assessment of fine motor skill predict successful central line insertion? Am J Surg 2016;212:573-578.

29. Bjerrum F, Strandbygaard J, Rosthøj S, Grantcharov T, Ottesen B, Sorensen JL. Evaluation of procedural simulation as a training and assessment tool in general surgery: simulating a laparoscopic appendectomy. J Surg Educ 2017;74:243-50.

30. Siddiqui NY, Galloway ML, Geller EJ, Green IC, Hur HC, Langston M, et al. Validity and reliability of the robotic objective structured assessment of technical skills. Obstet Gynecol 2014;123:1193-9.

31. Del Blanco Á, Torrente J, Fernández-Manjón B, Ruiz P, Giner M. Using a videogame to facilitate nursing and medical students' first visit to the operating theatre: a randomized controlled trial. Nurse Educ Today 2017;55:45-53.

32. Reisinger M. Der dimensional management prozess. ZEVrail 2015;133:160-3.

33. Byrne RM, Hoops HE, Herzig DO, Diamond SJ, Lu KC, Brasel KJ, et al. Assessing the value of endoscopy simulator modules designed to prepare residents for the fundamentals of endoscopic surgery examination. Dis Colon Rectum 2019;62:211-16.

34. Acton RD. The evolving role of simulation in teaching surgery in undergraduate medical education. Surg Clin North Am 2015;95:739-50.

35. Tillou X, Collon S, Martin-Francois S. Robotic surgery simulator : elements to build a training program. J Surg Educ 2016;73:870-8.

36. Kearney JA, Deutsch ES. Using simulation to improve systems. Otolaryngol Clin North Am 2017;50:1015-28.

37. Paige JT, Garbee DD, Brown KM, Rojas JD. Using simulation in interprofessional education. Surg Clin North Am 2015;95:751-66.

38. Fried MP, Uribe JI, Sadoughi B. The role of virtual reality in surgical training in otorhinolaryngology. Curr Opin Otolaryngol Head Neck Surg 2007;15:163-69.

39. Gardner AK, Scott DJ. Concepts for developing expert surgical teams using simulation. Surg Clin North Am 2015;95:717-28.

40. McKinley SK, Phitayakorn R. Emotional intelligence and simulation. Surg Clin North Am 2015;95:855-67.

41. Chauvin SW. Applying educational theory to simulation-based training and assessment in surgery. Surg Clin North Am 2015;95:695-715.

42. Evans $\mathrm{CH}$, Schenarts KD. Evolving educational techniques in surgical training. Surg Clin North Am 2016;96:71-88.

43. Cumin D, Boyd MJ, Webster CS, Weller JM. A systematic review of simulation for multidisciplinary team training in operating rooms. Simul Healthc 2013;8:171-9.

44. Miyasaka KW, Buchholz J, Lamarra D, Karakousis GC, Aggarwal R. Development and implementation of a clinical pathway approach to simulation-based training for foregut surgery. J Surg Educ 2015;72:625-35.

45. Sullivan SA, Bingman E, Rourke AO, Pugh CM. Piloting virtual surgical patient cases with 3rd-year medical students during the surgery rotation. Am J Surg 2016;211:689-96.

46. Stefanidis D, Sevdalis N, Paige J, Zevin B, Aggarwal R, Grantcharov T, et al. Simulation in surgery: 
what's needed next? Ann Surg 2015;261:846-853.

47. Pucher PH, Aggarwal R, Singh P, Srisatkunam T, Twaij A, Darzi A. Ward simulation to improve surgical ward round performance: a randomized controlled trial of a simulation-based curriculum. Ann Surg 2014;260:236-43.

48. Pucher PH, Darzi A, Aggarwal R. Simulation for ward processes of surgical care. Am J Surg 2013;206:96-102.

49. Evgeniou E, Loizou P. Simulation-based surgical education. ANZ J Surg. 2013;83:619-23.

50. Davies J, Khatib M, Bello F. Open surgical simulation: a review. J Surg Educ 2013;70:618-27.

51. Raphael RM, Hoops HE, Herzig DO, Diamond SJ, Lu KC. Assessing the value of endoscopy simulator modules designed to prepare residents for the fundamentals of endoscopic surgery examination. Dis Colon Rectum 2019;62:211-16.

52. Guo S, Cai X, Gao B. A tensor-mass method-based vascular model and its performance evaluation for interventional surgery virtual reality simulator. Int J Med Robot 2018;14:e1946.

53. Chen HE, Sonntag CC, Mirkin KA, Pepley DF, Han DC, Moore JZ, et al. From the simulation center to the bedside: validating the efficacy of a dynamic haptic robotic trainer in internal jugular central venous catheter placement. Am J Surg 2020;219:379-84.

54. Sanada Y, Hishikawa S, Katano T, Hirata Y, Yamada N, Okada N, et al. Surgical training and simulation of split-liver transplantation in an ex vivo porcine model. Liver Transpl 2019;25:510-14.

55. Schlottmann F, Long JM, Brown S, Patti MG. Low confidence levels with the robotic platform among senior surgical residents: simulation training is needed. J Robot Surg 2019;13:155-58.

56. Melich G, Pai A, Shoela R, Kochar K, Patel S, Parket J, et al. Rectal dissection simulator for da Vinci Surgery: details of simulator manufacturing with evidence of construct, face, and content validity. Dis Colon Rectum 2018;61:514-19.

57. Qiu K, Haghiashtiani G, McAlpine MC. 3D Printed organ models for surgical applications. Annu Rev Anal Chem 2018;11:287-306.

58. Groom P, Schofield L, Hettiarachchi N, Pickard S, Brown J, Sandars J, et al. Performance of emergency surgical front of neck airway access by head and neck surgeons, general surgeons, or anaesthetists: an in situ simulation study. Br J Anaesth 2019;123:696-703.

59. Moit H, Dwyer A, De Sutter M, Heinzel S, Crawford D. Standardized robotic training curriculum in a general surgery program. JSLS 2019;23:e2019.00045. 